\title{
Relationship between Disturbance of Micellar Formation and Steatorrhea
}

\author{
Makoto Ishikawa,* Osamu Masamune, Hiroshi Yamada, \\ Tsuneo Takahashi,* Shingo Matsuura, Koji Torinata, \\ Toshikatsu Gonal, Akio Nagasaki, Masao Tsubol and \\ Shoichi Yamagata \\ The Third Department of Internal Medicine, Tohoku University \\ School of Medicine, Sendai
}

Ishukawa, M., Masamune, O., Yamada, H., Takahashi, T., Matsudra, S., Torihata, K., Gonai, T., Nagasaki, A., Tsuboi, M. and Yamagata, S. Relationship between Disturbance of Micellar Formation and Steatorrhea. Tohoku J. exp. Med., 1976, 118 (Suppl.), 85-88 _- In order to clarify the correlation between decrease in micellar formation and degree of steatorrhea, upper intestinal content was analyzed after administering a test meal of Borgström et al. Fecal fat was also measured. It was demonstrated that micellar phase was composed chiefly of fatty acid and monoglyceride, with low concentrations of diglyceride and triglyceride. The fat composition of micellar phase was nearly constant in normal subjects and in cases of malabsorption syndrome. The correlation between the ratio of micellar fat to the whole ingested fat in the intestinal content and daily excretion of fecal fat was very marked. ___ micelle; steatorrhea; malabsorption syndromes

It has been known that conversion of dietary triglyceride to fatty acid and monoglyceride in a micellar phase is an essential step for the optimal transport of dietary fat across the intestinal mucosa. Therefore, it is conceivable that impairment of micelle formation causes a disturbance of intraluminal fat digestion with a consequence of steatorrhea. The purpose of our study is to clarify the correlation between decrease in micelle formation and degree of steatorrhea.

\section{Materials and Methods}

The present study was carried out on 12 healthy subjects and 20 patients with steatorrhea associated with malabsorption syndrome. The causes of the syndrome were chronic pancreatitis, pancreatectomy, gastrectomy and ileal diseases, each in five patients.

Intubation technique was applied to obtain the intestinal contents. Miller-Abbott tube was inserted into the proximal jejunum 20 to $30 \mathrm{~cm}$ beyond the ligament of Treitz and the position of the tube was monitored radiographically. A test meal by Borgström et al. (1957) consisting of $30 \mathrm{~g}$ of corn oil, $60 \mathrm{~g}$ of skimmed milk, $70 \mathrm{~g}$ of glucose and $400 \mathrm{ml}$ of water was administered for each test. The intestinal contents were kept in an ice box after being collected from the upper jejunum through a radio-opaque tube successively in four $30-\mathrm{min}$ samples for $2 \mathrm{hr}$. The frozen samples were quickly thawed and heated at $70^{\circ} \mathrm{C}$ for $10 \mathrm{~min}$ to inactivate pancreatic lipase before chemical analysis. To separate micelle from emulsion, $10 \mathrm{ml}$ of intestinal contents were centrifuged at $20,000 \times \mathrm{g}$ for $5 \mathrm{hr}$.

Received for publication, November 20, 1975.

* Present address: The Second Department of Internal Medicine, Yamagata University School of Medicine, Yamagata. 
An aliquot of clear infranatant solution of micellar fat was collected by needle aspiration, and fat from both micellar and total phases were extracted by Blankenhorn and Ahrens' method (1955). For a measurement of fat composition, the thin-layer chromatography with solvent system of heptane, ethylether and acetic acid $(50: 49: 1)$ was used. An analysis of fat was performed by the method of Amenta (1964). Fecal fat was measured by the method of van de Kamer et al. (1949).

\section{Results}

The percentage of the fat composition in micellar phase is shown in Fig. 1. The micellar phase was compcsed chiefly of fatty acid and monoglyceride with much lower concentrations of diglyceride and triglyceride, which were nearly constant in normal subjects and in cases of malabsorption syndrome.

The mean value of the ratio of fat content in micellar phase to the total fat content was $76.9 \pm 21.5 \%$ in 38 samples of normal subjects. It means that approximately 70 to $80 \%$ of administered fat is contained in micellar phase in the

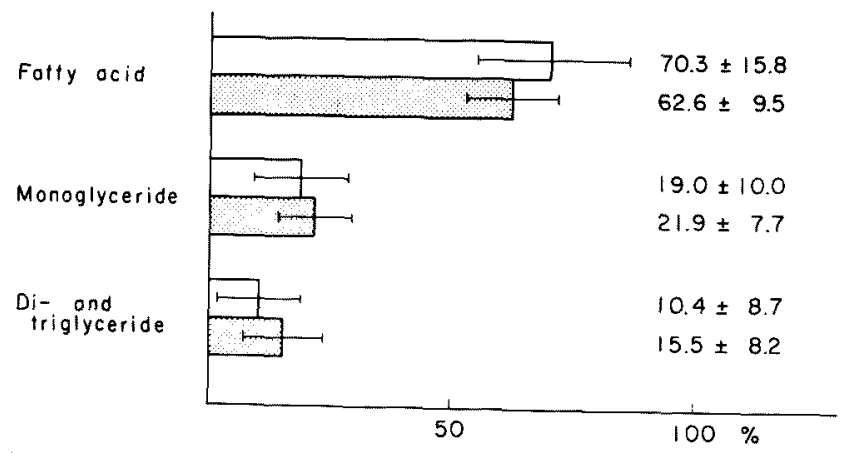

Fig. 1. Fat composition in micellar phase in normal subjects ( $\square$ ) and cases of malabsorp. tion syndrome $(\square)$.

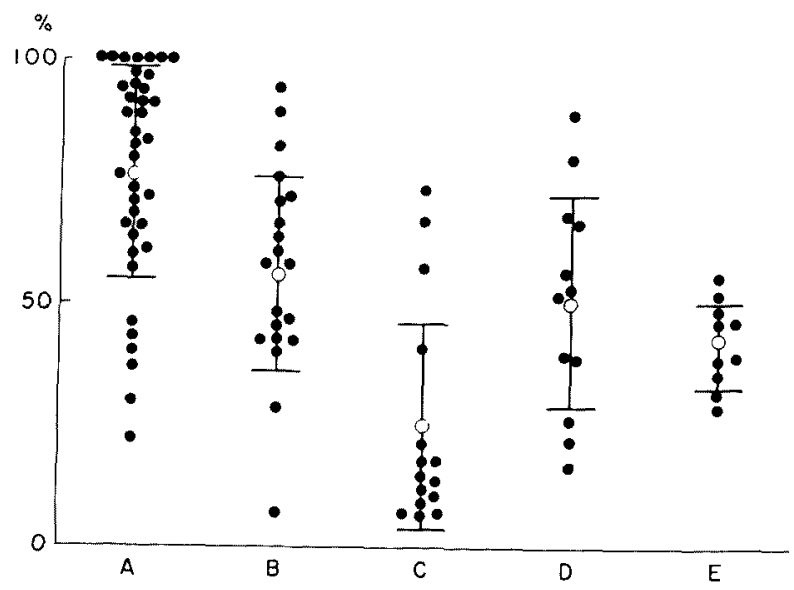

Fig. 2. Ratios of micellar fat to the total ingested fat in normal subjects (A), chronic pancreatitis (B), pancreatectomy (C), gastrectomy (D), and ileal diseases (E). 


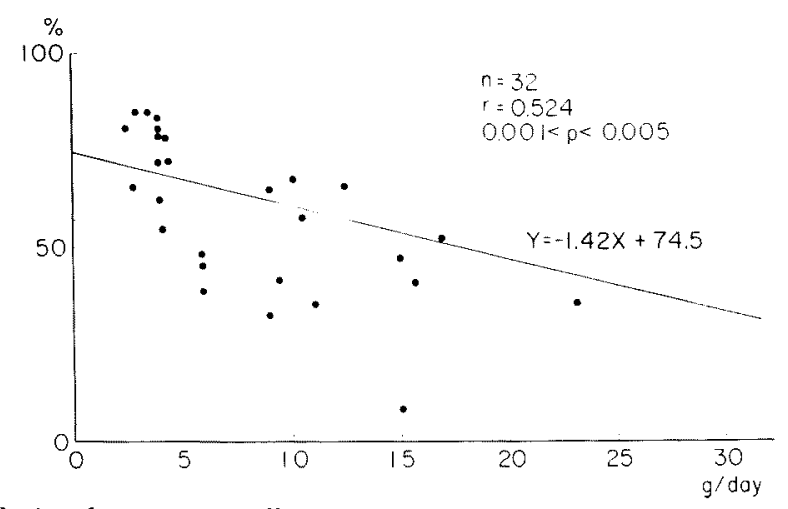

Fig. 3. Correlation between micellar fat and fecal fat in normal subjects and cases of various malabsorption syndromes.

upper intestinal contents of normal subjects. In contract, a decrease of micellar fat was shown in malabsorption syndrome, i.e., the ratio was $56.1 \pm 20.3 \%, 24.9 \pm$ $20.8 \%, 50.0 \pm 21.8 \%$ and $45.1 \pm 14.2 \%$ in cases of chronic pancreatitis, pancreatectomy, gastrectomy and ileal diseases, respectively (Fig. 2).

The correlation between micellar fat and fecal fat in normal subjects and cases of various malabsorption syndromes is demonstrated in Fig. 3. The correlation was remarkable, the correlation coefficient $r$ being 0.524 . It means that the daily excretion of $5 \mathrm{~g}$ of fecal fat corresponds to the intraluminal situation where micellar fat occupied $70 \%$ of the whole fat in the intestinal contents, and similarly $10 \mathrm{~g}$ of fecal fat excretion corresponds to $60 \%$ of the ingested fat in the micellar fat.

\section{Discussion}

Dietary fat consists predominantly of triglyceride of long-chain fatty acids that are insoluble in the intestinal content. Ingested fat is normally emulsified in the stomach before it is discharged into the small intestine. In the lumen of the upper small intestine, pancreatic lipase hydrolyzes triglyceride to fatty acids and 2-monoglyceride which are highly soluble in the bile salt micelle.

Bile salts are amphipathic molecules with asymmetric, non-polar and polar regions; the detergent property of these molecules depends on their formation of polymolecular aggregates called micelles, when their concentration in solution exceeds a critical level. The polar fats, fatty acid and monoglyceride are extremely soluble in the bile salt micelle, whereas non-polar fats are poorly soluble. From the percentage of the fat composition in micellar phase in our study, fatty acid and monoglyceride are chiefly contained in the micellar phase. The concentration of diglyceride and triglyceride was much lower and nearly constant in both normal subjects and cases of malabsorption syndromes. These results agree well with Hofmann and Borgström's (1964) findings obtained by separation with ultracentrifugation of intestinal contents. 
As dietary fat is absorbed from the micellar phase, the percentage of administered fat contained in micellar phase decides the degree of fat absorption into intestinal epithelial cells. In normal subjects, approximately 70 to $80 \%$ of administered fat is taken in micellar phase of the upper intestinal contents of normal subjects. In comparison with the ratio of fat in micellar phase to the total fat in normal subjects, its marked decrease was shown in cases of chronic pancreatitis, pancreatectomy, gastrectomy and ileal diseases.

It was shown that micellar fat and fecal fat in various malabsorption syndromes were well correlated. This study demonstrates that the decrease of the percentage of fat composed in micellar phase causes the increase of excretion of fecal fat; in other words, it demonstrates that the error of intraluminal digestion of fat is mainly responsible for malabsorption states in malabsorption syndromes commonly experienced in Japan.

\section{References}

1) Amenta, J.S. (1964) Rapid chemical method for quantification of lipids separated by thin-layer chromatography. J. Lipid Res., 5, 270-271.

2) Blankenhorn, D.H. \& Ahrens, E.D., Jr. (1955) Extraction, isolation, and identification of hydrolytic products of triglyceride digestion in man. J. biol. Chem., 212, 69-81.

3) Borgström, B., Dahlqvist, A., Lundh, G. \& Sjövall, J. (1957) Studies of intestinal digestion and absorption in the human. J. clin. Invest., 36, 1521-1536.

4) Hofmann, A.F. \& Borgström, B. (1964) The intraluminal phase of fat digestion in man, the lipid content of the micellar and oil phases of intestinal content obtained during fat digestion and absorption. J. clin. Invest., 43, 247-257.

5) van de Kamer, J.H., Huinink ten Bokkel \& Weyers, H.A. (1949) Rapid method for the determination of fat in feces. J. biol. Chem., 177, 347-355. 